\title{
Characterization of molecular signatures of supratentorial ependymomas
}

\author{
Matthew Torre ${ }^{1,2} \cdot$ Sanda Alexandrescu ${ }^{1,2} \cdot$ Adrian M. Dubuc $^{2,3} \cdot$ Azra H Ligon $^{2,3} \cdot$ Jason L. Hornick $\mathbb{B}^{2}$ • \\ David M. Meredith $\mathbb{1}^{2}$
}

Received: 16 April 2019 / Revised: 17 June 2019 / Accepted: 17 June 2019 / Published online: 2 August 2019

(c) United States \& Canadian Academy of Pathology 2019

\begin{abstract}
Ependymomas show poor correlation between World Health Organization grade and clinical outcome. A subgroup of supratentorial ependymomas are characterized by C11orf95-RELA fusions, presumed to be secondary to chromothripsis of chromosome 11, resulting in constitutive activation of the NF- $\mathrm{KB}$ signaling pathway and overexpression of cyclin D1, p65, and L1 cell adhesion molecule (L1CAM). These RELA-fused ependymomas are recognized as a separate, molecularly defined World Health Organization entity and might be associated with poor clinical outcome. In this study, we show that immunohistochemistry for NF-KB signaling components, such as L1CAM, p65, and cyclin D1, can help distinguish RELA-fused from non-RELA-fused supratentorial ependymomas. Furthermore, these three markers can reliably differentiate $R E L A$-fused ependymomas from a variety of histologic mimics. Lastly, we report that RELA-fused ependymomas may be associated with different chromosomal copy number changes and molecular alterations compared to their non-RELA-fused counterparts, providing additional insight into the genetic pathogenesis of these tumors and potential targets for directed therapies.
\end{abstract}

\section{Introduction}

Ependymomas are primary tumors of the central nervous system that arise along the ventricular system and spinal cord. In pediatric patients, ependymomas comprise $\sim 10 \%$ of primary central nervous system tumors, with the majority arising in the posterior fossa. In contrast, ependymomas account for $\sim 5 \%$ of adult primary central nervous system

Supplementary information The online version of this article (https:// doi.org/10.1038/s41379-019-0329-2) contains supplementary material, which is available to authorized users.

$\triangle$ David M. Meredith

dmmeredith@bwh.harvard.edu

1 Department of Pathology, Boston Children's Hospital and Harvard Medical School, Boston, MA, USA

2 Department of Pathology, Brigham and Women's Hospital and Harvard Medical School, Boston, MA, USA

3 Center for Advanced Molecular Diagnostics, Brigham and Women's Hospital and Harvard Medical School, Boston, MA, USA tumors, with the majority arising in the spinal cord and infratentorial region [1].

Differentiating World Health Organization grade II ependymomas from grade III ependymomas is generally made on the basis of histologic features such as elevated mitotic activity, necrosis, and vascular proliferation, but there are no strict criteria, and clinical outcome in patients is poorly correlated with tumor grade [1,2]. The discordance between World Health Organization grade and clinical outcome has been attributed, at least in part, to an underlying molecular heterogeneity within ependymomas. The most reproducible clinical prognostic factor is extent of total resection [3, 4]. Broad gains of chromosome 1q, particularly within the context of copy neutral genomes in posterior fossa ependymomas (i.e. group A ependymomas) [5], and losses of chromosome 9 are also associated with worse outcomes [6-10].

A recent study suggested that ependymomas could be classified into nine different subgroups based on their DNA methylation profiles [11]. One of the groups that emerged was characterized by recurrent $R E L A$ rearrangements, most often with the partner C11orf95. These ependymomas occurred exclusively in the supratentorial compartment and demonstrated chromothripsis of chromosome 11 and deletions of $C D K N 2 A$ [11]. Although initial studies showed that 
RELA-fused ependymomas are associated with poor clinical outcome [11, 12], subsequent studies have not confirmed the prognostic significance of RELA rearrangement as an independent marker [10, 12-15]. Regardless of its prognostic implications, RELA-fused ependymomas are recognized as a separate, genetically defined World Health Organization entity. Supratentorial ependymomas in the pediatric population not containing RELA rearrangements account for a smaller subset of tumors, often possessing rearrangements in $Y A P 1$ instead and few other recurrent alterations [11]. In contrast, non-RELA-fused ependymomas in adults remain poorly characterized.

In this study, we examine the genomic profiles of supratentorial ependymomas in a predominantly adult population in order to define recurrent alterations in nonRELA-rearranged tumors. Furthermore, we explore the immunophenotypes of supratentorial ependymomas and evaluate the sensitivity and specificity of $N F-\kappa B$ pathway components to accurately determine the presence of an underlying RELA rearrangement.

\section{Materials and methods}

The surgical pathology and consultation archives of Brigham and Women's Hospital (Boston, MA) were searched for cases of ependymoma (supratentorial, posterior fossa, and spinal) and histologic mimics that were diagnosed from 1990 to 2018. Histologic and immunohistochemical evaluation was performed for 122 cases: 16 supratentorial ependymomas, 21 posterior fossa ependymomas, 37 spinal cord ependymomas, 2 astroblastomas, 10 various neuroepithelial tumors with prominent angiocentric architecture, and 36 glioblastomas, including 22 with poorly differentiated or primitive neuronal components. Four of the supratentorial ependymomas were contributed by Boston Children's Hospital (Boston, MA).

\section{Histology and immunohistochemistry}

Hematoxylin- and eosin-stained sections of formalin-fixed, paraffin-embedded tissue were evaluated using routine light microscopy. Immunohistochemistry was performed on $5 \mu \mathrm{m}$ paraffin sections following routine heat antigen retrieval (10 mM sodium citrate buffer, $\mathrm{pH}$ 6.0). Nuclei were counterstained with hematoxylin. The primary antibodies used were cyclin D1 (1:250 dilution, clone SP4, Thermo Fisher Scientific, Waltham, MA), p65 (1:5000 dilution, clone D14E12, Cell Signaling, Danvers, MA), and L1CAM (1:2000 dilution, clone UJ127.11, Sigma, St. Louis, MO). For cyclin D1 and p65, positivity was defined as at least intermediate nuclear immunoreactivity in at least $50 \%$ of tumor cells to reduce false-positive cases secondary to nonspecific staining. L1CAM positivity was defined as intermediate or greater membranous immunoreactivity in $>5 \%$ of tumor cells. Fisher's exact test was used to make comparisons of immunohistochemical staining between groups. $p$ values $<0.05$ were considered statistically significant. Percentages were rounded to the nearest integer.

\section{Targeted exome sequencing}

The presence of genomic alterations was determined for all tumors with a histologic diagnosis of ependymoma (grade II or III) arising within the supratentorial compartment using a next-generation hybrid capture sequencing assay (Oncopanel) performed on genomic DNA isolated from formalinfixed, paraffin-embedded tissue. Oncopanel evaluates 447 genes and 191 introns across 60 genes. Structural rearrangements were detected by BreaKmer analysis, as previously described [16]. Calls by BreaKmer analysis were verified by manual review of the raw Binary Alignment Map files.

\section{Chromosomal microarray}

As part of the clinical diagnostic workup for the majority of supratentorial ependymoma cases, copy number analysis was performed using one of two microarray platforms: array comparative genomic hybridization using the $1 \times 1 \mathrm{M}$ Agilent SurePrint G3 Human Microarray (Santa Clara, CA) or single-nucleotide polymorphism array analysis using the Thermo Fisher Scientific Oncoscan Copy Number Variation assay. The Agilent assay uses 963,029 probes hybridized to reference DNA across the genome for an average of $\sim 2.1 \mathrm{~kb}$ between probes and $1.8 \mathrm{~kb}$ in the RefSeq track. Oncoscan is a single-nucleotide polymorphism-based array optimized for use with formalin-fixed, paraffin-embedded genomic DNA that detects both copy number changes and copy-neutral loss of heterozygosity, with $300 \mathrm{~kb}$ genome-wide resolution and $50-100 \mathrm{~kb}$ resolution within 900 oncogenes and tumor suppressor genes. For cases in which no microarray analysis was performed, copy number alterations were determined using the Oncopanel assay, which shows $86 \%$ sensitivity and $98 \%$ specificity compared with copy number determinations made using the Agilent $1 \times 1 \mathrm{M}$ microarray [16].

The study was conducted with Institutional Review Board approval (Brigham and Women's Hospital IRB 11-104).

\section{Results}

\section{Clinicopathologic characteristics of supratentorial ependymomas}

Of the 16 supratentorial ependymomas, 8 possessed RELA fusions (four males, four females; average age 19.3 years, range 
8-51 years), and all had originally been diagnosed as anaplastic ependymoma, World Health Organization grade III. Tumors largely involved the frontal and/or parietal lobes $(n=8)$, with one also involving the temporal lobe. One patient had two additional resections (7-month interval and a 14-month interval between surgeries) for recurrent/residual tumor.

The remaining 8 supratentorial ependymomas were negative for RELA fusions (four males, four females; average age 54.6 years, range 36-81 years), two of which were diagnosed as ependymoma, World Health Organization grade II, four of which were diagnosed as anaplastic ependymoma, World Health Organization grade III, and two of which were not graded. Tumors arose throughout the supratentorial compartment, with three arising in the temporal lobe, and one each occurring in the frontal lobe, parietal lobe, suprasellar region, lateral ventricle, and third ventricle. One patient had an additional resection (14-month interval between surgeries).

\section{Molecular profiles of RELA-fused and non-RELA- fused supratentorial ependymomas}

Eight of 16 supratentorial ependymomas exhibited C11orf95-RELA fusion events, with breakpoints occurring most often in intron 1 of RELA as previously described [17]. Within this group of tumors, frequent gains of $1 \mathrm{q}$ (5/8 cases; $63 \%)$ and deletions of $9 \mathrm{p}$ including $C D K N 2 A / B(100 \%)$ were the most common copy number alterations (Fig. 1). Chromothripsis involving $11 \mathrm{q}$ was present in only three cases $(38 \%)$, but focal single copy gains or losses involving the RELA locus were observed overall in $7 / 8$ tumors $(88 \%)$. Additional recurrent events included chromothripsis or losses of 10q (3/8 cases; $38 \%$ ) and losses on 22 (3/8 cases; $38 \%$ ). Genomic activation of both cyclin-dependent kinase and phosphoinositide 3-kinase pathways was evident via inactivating mutations in CDKN2A (2/8 cases; 25\%), CDKN1C (1/8 cases, 13\%), PIK3RI (1/8 cases; 13\%), and
Fig. 1 Summary of clinicopathologic features, NF-кB component immunohistochemistry, and molecular profiles of supratentorial ependymomas

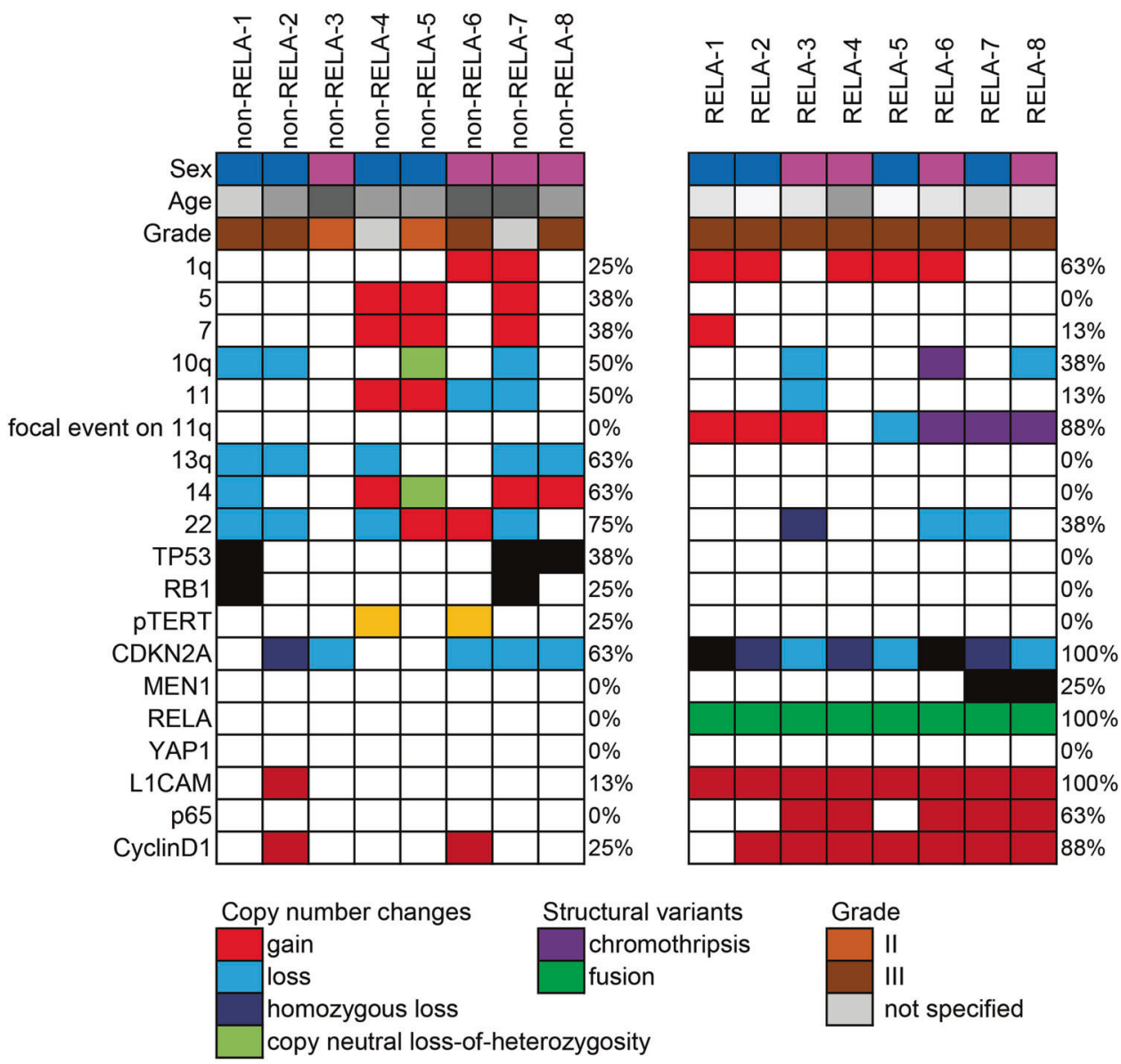

Single nucleotide variants gain-of-function loss-of-function

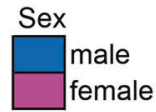

Immunohistochemistry positive
Age of Initial Diagnosis

0-9 years

10-19 years

20-39 years

$40-59$ years

$60+$ years 

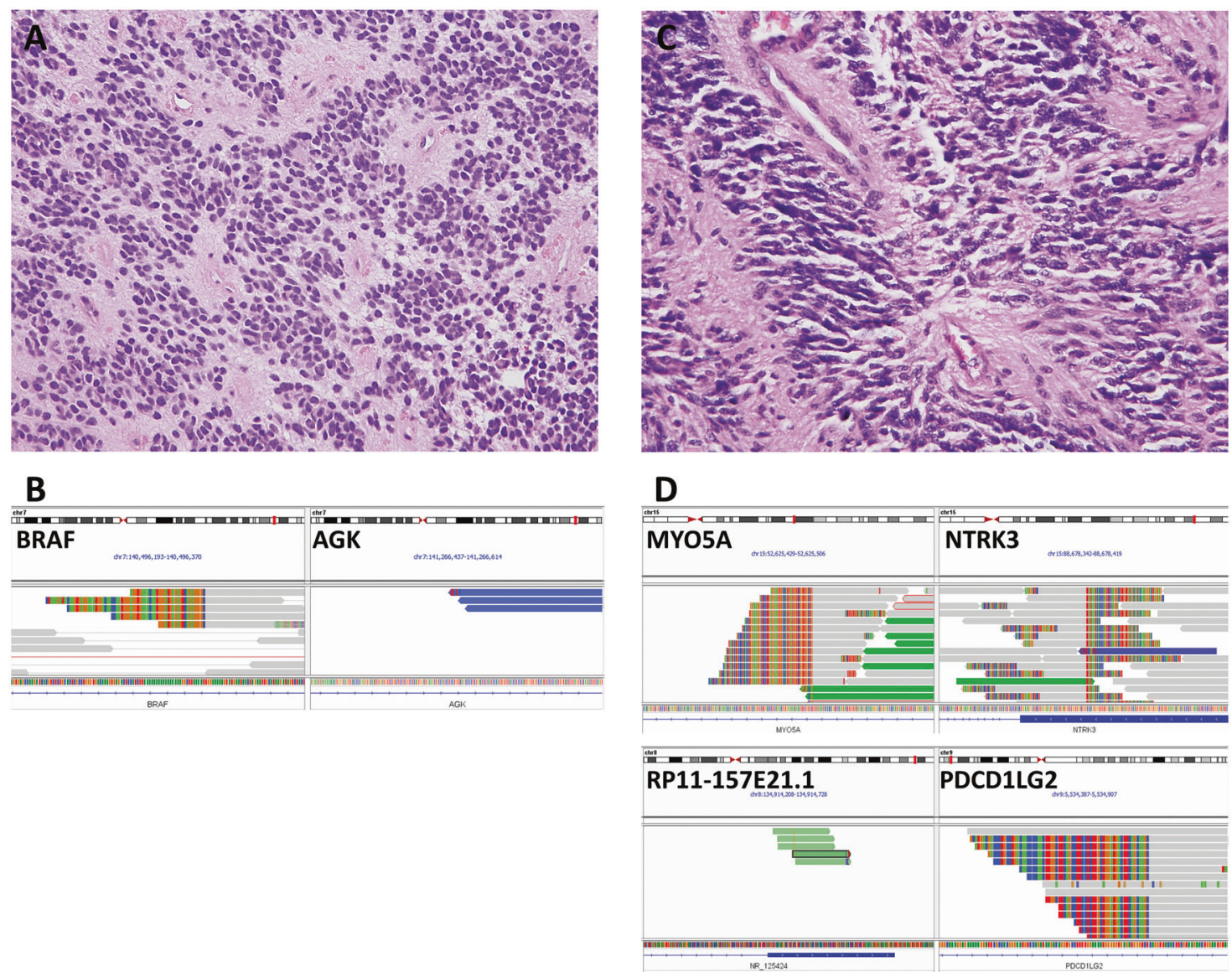

Fig. 2 Supratentorial ependymomas with potentially targetable structural rearrangements included a $R E L A$-fused ependymoma (a) with an $A G K$ $B R A F$ fusion (b), and a non-RELA-fused ependymoma (c) with MYO5A-NTRK3 and RP11-157E21.1-PDCD1LG2 fusions (d)

PTEN (1/8 cases; 13\%). Intriguingly, two cases showed heterozygous truncating nonsense mutations in MEN1; however, neither patient has thus far exhibited stigmata of multiple endocrine neoplasia (MEN type I) syndrome. Additional novel events included single instances of an activating $A G K-B R A F$ fusion (Fig. 2) and a missense NTRK1 p.R748Q variant occurring in the kinase domain.

In contrast, non-RELA-fused tumors showed fewer gains of $1 \mathrm{q}$ ( $2 / 8$ cases; $25 \%$ ), fewer deletions of $9 \mathrm{p}$ ( $5 / 8$ cases; $63 \%$ ), and were more likely to demonstrate multiple wholechromosome alterations. Unique to this subgroup were broad deletions of chromosome 13 (5/8 cases; 63\%), polysomy 5 (3/8 cases; $38 \%$ ), and monosomy/polysomy 14 (4/8 cases; 50\%). Polysomy $7(38 \%$ versus $13 \%)$ and alterations of $22 \mathrm{q}(75 \%$ versus $38 \%)$ were both more frequent in non-RELA-fused ependymomas versus RELAfused ependymomas. Mutations in TERT promoter (2/8 cases; $25 \%), T P 53$ (3/8 cases; $38 \%$ ), and RBI (2/8 cases; $25 \%)$ were exclusively observed in non-RELA-fused ependymomas. One case showed chromothripsis of chromosome 15 with multiple structural rearrangements, including
MYO5A-NTRK3, RP11-157E21.1-PDCD1LG2, and USP8$A D P G K$. Representative images of the tumors harboring MYO5A-NTRK3 and RP11-157E21.1-PDCD1LG2 fusions are shown in Fig. 2. TMEFF2-FOXO1 and PCGF1$C R E B B P$ structural variants were also observed in the non-RELA-fused cohort. Importantly, no YAPl fusions were detected in any of the 16 ependymomas above.

A comprehensive description of molecular aberrations observed in our group of supratentorial ependymomas is detailed in Supplementary Tables 1-3.

Of note, three cases that had been initially diagnosed as ependymoma based on presence of classic histologic features were reclassified based on characteristic genomic findings (Fig. 3). One tumor showed a focal homozygous deletion of SMARCB1 and few other alterations, permitting reclassification as atypical teratoid/rhabdoid tumor. Two other cases showed classic glioblastoma alterations (i.e. polysomy 7 , monosomy $10, T E R T$ promoter mutation), one of which also harbored the oncogenic FGFR3-TACC3 fusion. None of these tumors demonstrated the presence of fusions in either RELA or YAPI. 

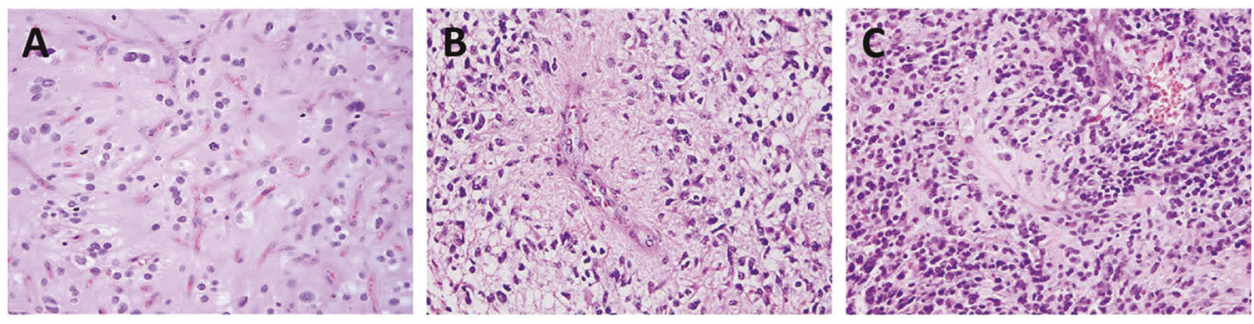

Fig. 3 Tumors reclassified as other entities following molecular testing included a glioblastoma with FGFR3-TACC3 fusion (a), a glioblastoma showing prominent perivascular arrangement of tumor cells (b) with conventional genomic alterations (polysomy 7, monosomy 10, TERT promoter mutation), and an atypical teratoid/rhabdoid tumor with focal homozygous deletion of SMARCB1 (c)

Table 1 Summary of NF- $\mathrm{KB}$ component immunohistochemistry

\begin{tabular}{lcccc}
\hline Tumor type & Cyclin D1 (\%) & p65 (\%) & L1CAM (\%) & $\begin{array}{c}\text { Combinatorial } \\
\text { immunohistochemistry (\%) }\end{array}$ \\
\hline RELA-fused supratentorial ependymoma & $7 / 8(88 \%)$ & $5 / 8(63 \%)$ & $8 / 8(100 \%)$ & $7 / 8(88 \%)$ \\
Non-RELA-fused supratentorial ependymoma & $2 / 8(25 \%)$ & $0 / 8(0 \%)$ & $1 / 8(13 \%)$ & $1 / 8(13 \%)$ \\
Posterior fossa ependymoma & $1 / 21(5 \%)$ & $0 / 21(0 \%)$ & $0 / 21(0 \%)$ & $0 / 21(0 \%)$ \\
Spinal ependymoma & $0 / 37(0 \%)$ & $0 / 37(0 \%)$ & $0 / 37(0 \%)$ & $0 / 37(0 \%)$ \\
Ependymoma mimics (total) & & & $1 / 48(2 \%)$ \\
Astroblastomas & $2 / 48(4 \%)$ & $1 / 48(2 \%)$ & $3 / 48(6 \%)$ & $0 / 2(0 \%)$ \\
Neuroepithelial tumors with angiocentric architecture & $1 / 10(10 \%)$ & $1 / 10(10 \%)$ & $0 / 10(0 \%)$ & $1 / 10(10 \%)$ \\
Glioblastomas & $1 / 14(7 \%)$ & $0 / 14(0 \%)$ & $1 / 14(7 \%)$ & $0 / 14(0 \%)$ \\
Glioblastomas with poorly differentiated or primitive & $0 / 22(0 \%)$ & $0 / 22(0 \%)$ & $2 / 22(9 \%)$ & $0 / 22(0 \%)$ \\
neuronal components & & & $0 / 2(0 \%)$ & $0 / 2(0 \%)$
\end{tabular}

${ }^{a}$ Mimics of ependymoma include astroblastoma, neuroepithelial tumors with angiocentric architecture, and glioblastomas, including those with poorly differentiated or primitive neuronal components

\section{Immunohistochemistry for NF-KB components}

In order to determine the sensitivity and specificity of NF$\kappa \mathrm{B}$ pathway components at detecting RELA rearrangements, we performed immunohistochemistry with the markers L1CAM, p65, and cyclin D1 in ependymomas from all anatomic locations, as well as in several additional entities in the differential diagnosis (summarized in Table 1).

Cyclin D1 was positive in 7 of 8 RELA-fused ependymomas (88\%), 2 of 8 non-RELA-fused ependymomas (25\%; $p<0.05), 1$ of 21 posterior fossa ependymomas $(5 \%$; $p<0.001), 1$ of 14 glioblastomas with conventional histology $(7 \% ; p<0.001)$, and 1 of 10 neuroepithelial tumors with angiocentric architecture $(10 \% ; p<0.005)$, with immunopositivity occurring in an atypical teratoid/rhabdoid tumor. None of the 37 spinal cord ependymomas $(0 \%$; $p<0.001), 2$ astroblastomas ( $0 \%)$, or 22 glioblastomas with poorly differentiated or primitive neuronal components $(0 \% ; p<0.001)$ were immunopositive. Collectively, 2 of 48 histologic mimics were immunopositive for cyclin D1 (4\%; $p<0.001)$. When comparing the immunophenotype of $R E L A$ versus non-RELA-fused supratentorial ependymomas (Fig. 1, Table 1), cyclin D1 had a sensitivity of $88 \%$, specificity of $75 \%$, positive predictive value of $78 \%$, and negative predictive value of $86 \%$ for detecting RELA fusions.

P65 was positive in 5 of 8 RELA-fused ependymomas (63\%), and 1 of 10 neuroepithelial tumors with angiocentric architecture $(10 \% ; p<0.05)$, with immunopositivity again occurring in an atypical teratoid/rhabdoid tumor. None of the 8 non-RELA-fused ependymomas ( $0 \% ; p<0.05), 21$ posterior fossa ependymomas $(0 \% ; p<0.001), 37$ spinal cord ependymomas $(0 \% ; p<0.001), 2$ astroblastomas $(0 \%)$, or 36 glioblastomas $(0 \% ; p<0.001)$ were immunopositive. Collectively, 1 of 48 histologic mimics were immunopositive for p65 $(2 \% ; p<0.001)$. When comparing the immunophenotype of $R E L A$ versus non-RELA-fused supratentorial ependymomas (Fig. 1, Table 1), p65 had a sensitivity of 63\%, specificity of $100 \%$, positive predictive value of $100 \%$, and negative predictive value of $73 \%$ for detecting RELA fusions.

L1CAM was positive in all 8 RELA-fused ependymomas (100\%), 1 of 8 non-RELA-fused ependymomas (13\%; $p<0.005), 2$ of the 22 glioblastomas with poorly differentiated or primitive neuronal components $(9 \% ; p<0.001)$, and 1 of 14 glioblastomas with conventional histology (7\%; $p<0.001)$. None of the 21 posterior fossa ependymomas $(0 \% ; p<0.001), 37$ spinal cord ependymomas $(0 \%$; $p<0.001), 2$ astroblastomas $(0 \%)$, or 10 neuroepithelial 

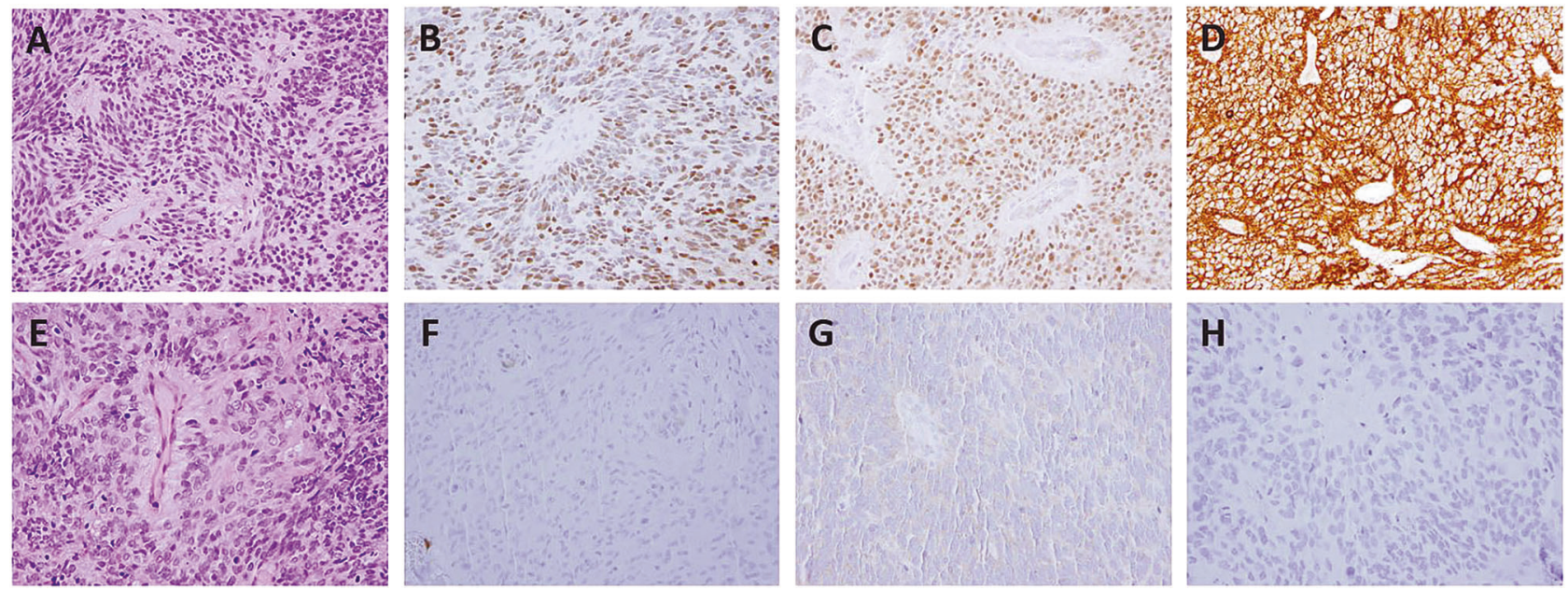

Fig. 4 Representative image of a RELA-fused supratentorial ependymoma (a) with positive immunostaining for (b) cyclin D1 (strong diffuse nuclear positivity), (c) p65 (strong diffuse nuclear positivity),

and (d) L1CAM. Representative image of a non-RELA-fused supratentorial ependymoma (e) with negative immunostaining for cyclin D1 (f), p65 (g), and L1CAM (h)

Fig. 5 Evaluated histologic mimics of ependymoma included astroblastomas (a), various neuroepithelial tumors with angiocentric architecture (b), and (c) glioblastomas, including those with poorly differentiated or primitive neuronal components (d)
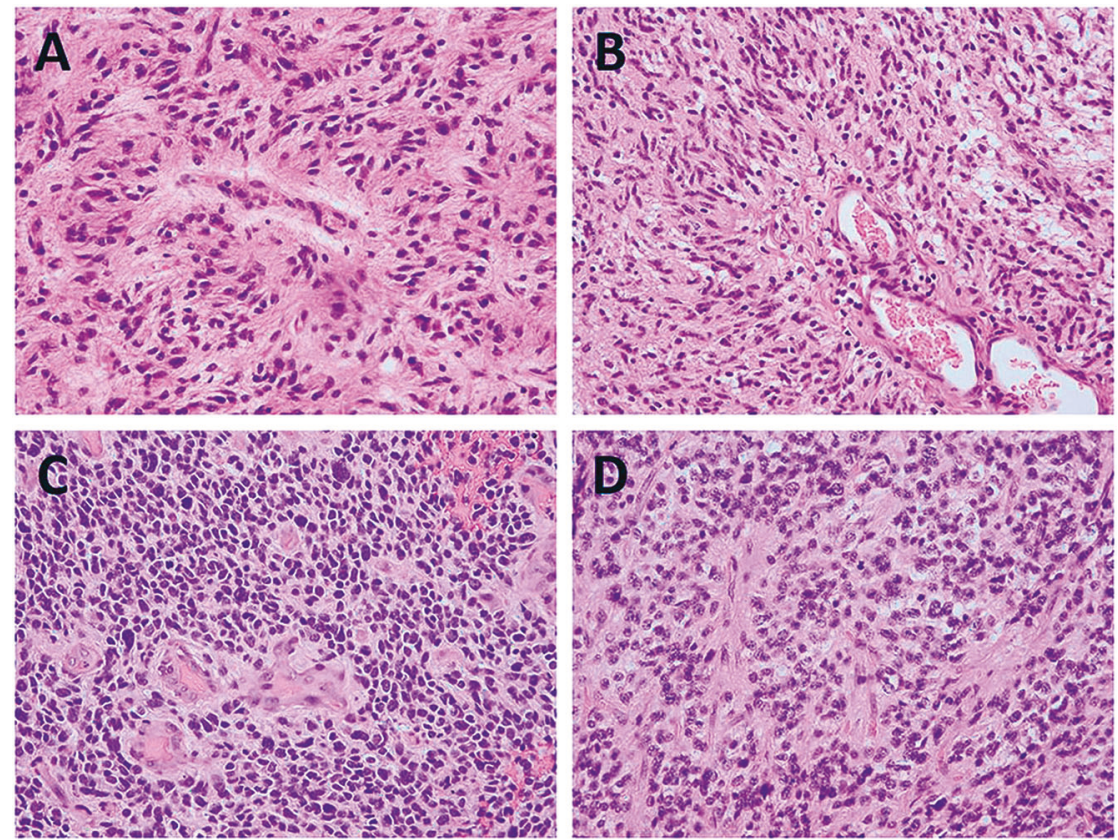

tumors with angiocentric architecture $(0 \% ; p<0.001)$ were immunopositive. Collectively, 3 of 48 histologic mimics were immunopositive for L1CAM $(6 \% ; p<0.001)$. When comparing the immunophenotype of RELA versus nonRELA-fused supratentorial ependymomas (Fig. 1, Table 1), L1CAM had a sensitivity of $100 \%$, specificity of $88 \%$, positive predictive value of $89 \%$, and negative predictive value of $100 \%$ for detecting RELA fusions. Figure 4 shows representative histology and NF- $\mathrm{KB}$ component immunohistochemistry from a RELA-fused ependymoma and a nonRELA-fused ependymoma. Representative histology from select histologic mimics of ependymomas are shown in Fig. 5.
The utility of combinatorial immunohistochemistry, in which a positive result is defined as at least two of three immunostains being positive, was also assessed. Combinatorial immunohistochemistry was positive in 7 of 8 $R E L A$-fused ependymomas (88\%), 1 of 8 non-RELA-fused ependymomas $(13 \% ; p<0.05)$, and 1 of 10 neuroepithelial tumors with angiocentric architecture $(10 \% ; p<0.005)$, with immunopositivity occurring in an atypical teratoid/ rhabdoid tumor. None of the posterior fossa ependymomas $(0 \% ; p<0.001)$, spinal cord ependymomas $(0 \% ; p<0.001)$, astroblastomas $(0 \%)$, or glioblastomas $(0 \% ; p<0.001)$ were immunopositive. Collectively, 1 of 48 histologic mimics was immunopositive with a combinatorial 
immunohistochemical approach $(2 \% ; p<0.001)$. When comparing the immunophenotype of RELA- versus nonRELA-fused supratentorial ependymomas, combinatorial immunohistochemistry had a sensitivity of $88 \%$, specificity of $88 \%$, positive predictive value of $88 \%$, and negative predictive value of $88 \%$ for detecting RELA fusions. While a combinatorial approach did not improve the ability to differentiate RELA versus non-RELA-fused supratentorial ependymomas compared to L1CAM alone, it did reduce the number of positive cases among the histologic mimics.

Two patients with supratentorial ependymomas had multiple resections for recurrent/residual tumor, and the cyclin D1, p65, and L1CAM immunophenotype did not change over time.

\section{Discussion}

RELA-fused ependymomas, which are recognized as a separate World Health Organization entity, arise exclusively in the supratentorial compartment, drive tumorigenesis via aberrant NF- $\mathrm{NB}$ signaling, and might be associated with a worse prognosis compared to their non-RELA-fused counterparts. No gold standard or guidelines exist for demonstrating the presence of these fusions. Other than comprehensive RNA sequencing or whole-genome sequencing, it has been challenging to identify these fusions in clinical assays available to many pathologists. Fluorescence in situ hybridization with custom break-apart probes has been used in the research setting to detect C11orf95-RELA fusions [15, 17], but clinical implementation may be challenging due to the difficulty of interpreting the assay, as well as the limited shelf life of fluorescently labeled probes. In addition, break-apart fluorescence in situ hybridization may result in false-negative or false-positive results depending on the target sequence of the probes. We recommend that an immunohistochemistry panel of NF- $\mathrm{NB}$ signaling components be performed for the initial workup of supratentorial ependymomas. The panel of immunostains should be particularly useful for pediatric supratentorial ependymomas, as the incidence of RELA fusions is $~ 70 \%$ in pediatric cases compared to $20 \%$ in adult cases $[12,17]$.

This study builds on prior work by demonstrating that immunohistochemistry for NF- $\mathrm{NB}$ components shows differential immunostaining in RELA-fused ependymomas compared to non-RELA-fused ependymomas. Gessi et al., for instance, reported that L1CAM had a sensitivity of $94 \%$ and specificity of $76 \%$ for detecting RELA fusions, whereas p65 had a sensitivity of $100 \%$ and a specificity of $92 \%$ [14]. In our cohort, L1CAM had a sensitivity of $100 \%$ and specificity of $88 \%$, p65 had a sensitivity of $63 \%$ and specificity of $100 \%$, and cyclin D1 had a sensitivity of $88 \%$ and specificity of $75 \%$. Immunohistochemistry for all three $N F-\kappa B$ signaling components reliably distinguished these two groups of ependymomas. The variation in performance of these markers across studies suggests that a panel of immunostains should be used as an initial screen for the RELA fusion rather than any single marker. It is unclear why in our study p65 had a lower sensitivity for detecting the RELA-fusion compared to that reported in the literature [14], especially given the presumed pathogenic mechanism of the RELA fusion and the strong correlation between the presence of RELA fusions and overexpression of p65 [17].

RELA fusions can occur in the absence of chromothripsis of chromosome 11. Immunohistochemistry for $N F-\kappa B$ components appears to be a more sensitive surrogate marker than chromosomal copy number changes at detecting this fusion event. In our cohort, chromothripsis of chromosome 11 was identified in only 3 of 8 cases and should therefore not be used to exclude a diagnosis of RELA-fused ependymoma.

Our study adds to the literature by demonstrating that immunohistochemistry for proteins upregulated by RELA fusions can differentiate this molecular subtype of supratentorial ependymoma from a variety of histologic mimics, such as neuroepithelial tumors with angiocentric architecture and glioblastomas. Cyclin D1, p65, and L1CAM were each able to reliably distinguish RELA-fused ependymomas from histologic mimics, and a combinatorial immunohistochemical approach outperformed individual antibodies against cyclin D1 and p65.

Furthermore, as anticipated, ependymomas arising from non-supratentorial compartments (i.e. posterior fossa and spinal cord) did not show immunopositivity for these three markers, with one exception of a posterior fossa ependymoma with isolated cyclin D1 immunoreactivity. This general observation, which is consistent with molecular data demonstrating that RELA-fusions occur exclusively in supratentorial ependymomas [11], could potentially be of clinical utility when trying to ascertain the site of primary tumor in the setting of ependymoma that has spread to multiple anatomic compartments.

However, a few exceptions to the expected immunostaining patterns should be mentioned. A number of nonRELA-fused tumors in our study showed unanticipated positivity for one or more markers. One case was an atypical teratoid/rhabdoid tumor that was positive for cyclin D1 and p65 but negative for L1CAM. Loss of tumor suppressor SMARCBI/INI1 may result in de-repression of cyclin D1 [18], and overexpression of cyclin D1 has indeed been reported in atypical teratoid/rhabdoid tumors [19]. In addition, there were three glioblastomas that were positive for L1CAM but negative for cyclin D1 and p65. A minority of glioblastomas have been reported in the literature as having aberrant expression of L1CAM, which is associated with increased resistance to radiation and chemotherapy as 
well as higher proliferation and tumor cell motility/infiltration [20-22]. L1CAM positivity in the absence of concurrent cyclin D1 and p65 positivity suggests that upregulation of L1CAM may occur through mechanisms independent of constitutive NF- $\mathrm{\kappa B}$ activation, such as aberrant Wnt signaling, among other pathways [23]. Moreover, one non-RELA-fused supratentorial ependymoma was positive for L1CAM and cyclin D1 but negative for p65. Although no RELA fusion event was identified, we cannot exclude the possibility that this tumor contained an alternate C11 orf95 or RELA rearrangement not captured by our assay.

Chromothripsis involving chromosome 11, losses involving chromosomes 3, 9 (often resulting in homozygous loss of $C D K N 2 A$ ), 10, and 22, and gain of chromosome 1q have been reported as frequent copy number alterations in RELA-fused ependymomas, while non-RELAfused ependymomas-specifically those with $Y A P 1$ fusions -are reported to have few copy number changes other than focal aberrations on chromosome 11q and loss of 22q [11]. The genetic profile of supratentorial ependymomas molecularly confirmed negative for both RELA and YAPI fusions are not well characterized. However, $B C O R$ tandem duplication and fusions involving EP300-BCORL1 and FOXO1STK24, as well as MAML2-ASCL2 and MARK2-ADCY3, have been described $[13,15]$. Here we demonstrate that RELA-fused ependymomas have distinct copy number changes and single nucleotide variants compared to their non-RELA-fused supratentorial ependymoma counterparts (apart from the C11orf95-RELA fusion event). The genetic profile of our cohort of supratentorial ependymomas negative for RELA and YAPl fusions contributes to the increasing recognition that this group of tumors is molecularly heterogeneous, with a subset of cases possessing mutations in TP53, RBI, and the TERT promoter region [13]. Of note, none of our RELA-fused ependymomas demonstrated mutations in these genes or regions of interest, but a subset of cases had loss-of-function variants in $M E N 1$. Furthermore, in comparison to RELA-fused ependymomas, non-RELA-fused supratentorial ependymomas tended to have more complex copy number changes, including numerous polysomies. Gain of 1q, loss of $10 \mathrm{q}$ (PTEN), loss of CDKN2A, monosomy 22, and NTRK aberrations were seen in both RELA-fused and non-RELAfused supratentorial ependymomas.

A number of compelling structural rearrangements were also observed that may provide insight into the molecular pathogenesis of these tumors and serve as targets for directed therapies. The non-RELA-fused cohort contained TMEFF2-FOXO1, MYO5A-NTRK3, and RP11-157E21.1$P D C D 1 L G 2$ rearrangements, while a single RELA-fused tumor showed an additional $A G K-B R A F$ rearrangement. FOXO1 is a member of the forkhead family of transcription factors that mediates numerous cellular processes, including cell survival, apoptosis, and cellular differentiation, and drives oncogenesis in a subset of rhabdomyosarcomas [24]. Also reported in a recent study of supratentorial ependymomas, FOXO1 fusions may be a recurrent driver event in a subset of non-RELA, non-YAPl-fused tumors [13]. NTRK fusions are seen across multiple tumor types, including both pediatric and adult gliomas, and have shown promising clinical response to Trk inhibitors [25-27]. MYO5A-NTRK3 fusions in particular have been described previously in melanocytic neoplasms and show constitutive upregulation of mitogen-activated protein kinase, phosphoinositide 3-kinase, and phospholipase $\mathrm{C} \gamma 1$ pathways [28-30]. PDCD1LG2 encodes PD-L2, the overexpression of which has been linked to response to PD-1 blockade inhibition [31]. AGK-BRAF fusions have been reported in several tumor types, including pleomorphic xanthoastrocytoma, anaplastic oligodendroglioma, lung adenocarcinoma, melanoma, and papillary thyroid carcinoma [32,33], and there is preliminary evidence that tumors with $A G K-B R A F$ fusions may respond to tyrosine kinase inhibitor targeted therapy [32, 34].

Multiple loss-of-function mutations involving MEN1 were seen in the RELA-fused cohort. Although ependymomas have been linked to MEN1 syndrome [35], neither of the patients whose tumors contained these mutations have thus far demonstrated presence of endocrine neoplasia, likely a result of heterozygous, somatic inactivation of the gene. Nonetheless, the presence of MEN1 alterations in RELA-fused ependymoma cases-and absence in nonRELA-fused cases-may implicate an important role of MEN1 in the pathogenesis of these tumors. MEN1 encodes menin, a tumor suppressor that interacts with p65 to repress NF-KB signaling, possibly via Sirt1-mediated deacetylation of p65 [36, 37]. Additional work, however, is needed to determine the possible prognostic role of $M E N 1$ alterations in RELA-fused ependymomas.

In summary, supratentorial ependymomas have distinct molecular profiles depending on RELA fusion status, with non-RELA-fused tumors being enriched for mutations involving TP53, RB1, and the TERT promoter and possessing generally more complex copy number changes, particularly multiple polysomies. RELA-fused tumors, in contrast, have recurrent genetic aberrations involving $M E N 1$ in a subset of tumors. Regardless of RELA status, supratentorial ependymomas not infrequently possess targetable fusion events. Furthermore, immunohistochemistry for NF$\kappa \mathrm{B}$ components, particularly L1CAM and $\mathrm{p} 65$, is a useful initial diagnostic step to distinguish RELA from non-RELAfused supratentorial ependymomas. In addition, multiple NF-kB markers, including cyclin D1, p65, and L1CAM, can be used to differentiate RELA-fused ependymomas from a variety of histologic mimics of ependymoma. 
Acknowledgements We would like to acknowledge the staff members of the Histology Laboratory and the Center for Advanced Molecular Diagnostics at Brigham and Women's Hospital for their contributions and support.

Author contributions MT and DMM drafted the manuscript. All authors contributed to the concept and design of the study, the acquisition, interpretation, or analysis of the data, and the critical revision of the manuscript.

\section{Compliance with ethical standards}

Conflict of interest The authors declare that they have no conflict of interest.

Publisher's note: Springer Nature remains neutral with regard to jurisdictional claims in published maps and institutional affiliations.

\section{References}

1. Ellison DW, McLendon R, Wiestler OD, Kros JM, Korshunov A, $\mathrm{Ng} \mathrm{H}-\mathrm{K}$, et al. Ependymal tumors. In: Louis DN, Ohgaki H, Wiestler OD, Cavenee WK, Ellison DW, Figarella-Branger D, Perry A, Reifenberger G, von Deimling A, editors. WHO classification of tumours of the central nervous system. Revised 4th ed. Lyon: International Agency for Research on Cancer; 2016. p. 101-14.

2. Ellison DW, Kocak M, Figarella-Branger D, Felice G, Catherine G, Pietsch T, et al. Histopathological grading of pediatric ependymoma: reproducibility and clinical relevance in European trial cohorts. J Negat Results Biomed. 2011;10:7.

3. Figarella-Branger D, Civatte M, Bouvier-Labit C, Gouvernet J, Gambarelli D, Gentet JC, et al. Prognostic factors in intracranial ependymomas in children. J Neurosurg. 2000;93:605-13.

4. Merchant TE, Li C, Xiong X, Kun LE, Boop FA, Sanford RA. Conformal radiotherapy after surgery for paediatric ependymoma: a prospective study. Lancet Oncol. 2009;10:258-66.

5. Witt H, Mack SC, Ryzhova M, Bender S, Sill M, Isserlin R, et al. Delineation of two clinically and molecularly distinct subgroups of posterior fossa ependymoma. Cancer Cell. 2011;20:143-57.

6. Yang I, Nagasawa DT, Kim W, Spasic M, Trang A, Lu DC, et al. Chromosomal anomalies and prognostic markers for intracranial and spinal ependymomas. J Clin Neurosci. 2012;19:779-85.

7. Pajtler KW, Wen J, Sill M, Lin T, Orisme W, Tang B, et al. Molecular heterogeneity and CXorf67 alterations in posterior fossa group A (PFA) ependymomas. Acta Neuropathol. 2018;136:211-26.

8. Araki A, Chocholous M, Gojo J, Dorfer C, Czech T, Heinzl H, et al. Chromosome 1q gain and tenascin-C expression are candidate markers to define different risk groups in pediatric posterior fossa ependymoma. Acta Neuropathol Commun. 2016;4:88.

9. Godfraind C, Kaczmarska JM, Kocak M, Dalton J, Wright KD, Sanford RA, et al. Distinct disease-risk groups in pediatric supratentorial and posterior fossa ependymomas. Acta Neuropathol. 2012;124:247-57.

10. Merchant TE, Bendel AE, Sabin ND, Burger PC, Shaw DW, Chang E, et al. Conformal radiation therapy for pediatric ependymoma, chemotherapy for incompletely resected ependymoma, and observation for completely resected, supratentorial ependymoma. J Clin Oncol. 2019;37:974-83.

11. Pajtler KW, Witt H, Sill M, Jones DT, Hovestadt V, Kratochwil F, et al. Molecular classification of ependymal tumors across all CNS compartments, histopathological grades, and age groups. Cancer Cell. 2015;27:728-43.
12. Malgulwar PB, Nambirajan A, Pathak P, Faruq M, Rajeshwari M, Singh M, et al. C11orf95-RELA fusions and upregulated NF-KB signalling characterise a subset of aggressive supratentorial ependymomas that express L1CAM and nestin. J Neurooncol. 2018;138:29-39.

13. Fukuoka K, Kanemura Y, Shofuda T, Fukushima S, Yamashita S, Narushima D, et al. Significance of molecular classification of ependymomas: C11orf95-RELA fusion-negative supratentorial ependymomas are a heterogeneous group of tumors. Acta Neuropathol Commun. 2018;6:134.

14. Gessi M, Giagnacovo M, Modena P, Elefante G, Gianno F, Buttarelli FR, et al. Role of immunohistochemistry in the identification of supratentorial C11ORF95-RELA fused ependymoma in routine neuropathology. Am J Surg Pathol. 2019;43:56-63.

15. Pages M, Pajtler KW, Puget S, Castel D, Boddaert N, TauziedeEspariat A, et al. Diagnostics of pediatric supratentorial RELA ependymomas: integration of information from histopathology, genetics, DNA methylation and imaging. Brain Pathol. 2019;29:325-35.

16. Garcia EP, Minkovsky A, Jia Y, Ducar MD, Shivdasani P, Gong $\mathrm{X}$, et al. Validation of OncoPanel: a targeted next-generation sequencing assay for the detection of somatic variants in cancer. Arch Pathol Lab Med. 2017;141:751-8.

17. Parker M, Mohankumar KM, Punchihewa C, Weinlich R, Dalton $\mathrm{JD}, \mathrm{Li} \mathrm{Y}$, et al. C11orf95-RELA fusions drive oncogenic NFkappaB signalling in ependymoma. Nature. 2014;506:451-5.

18. Zhang ZK, Davies KP, Allen J, Zhu L, Pestell RG, Zagzag D, et al. Cell cycle arrest and repression of cyclin D1 transcription by INI1/hSNF5. Mol Cell Biol. 2002;22:5975-88.

19. Fujisawa H, Misaki K, Takabatake Y, Hasegawa M, Yamashita J. Cyclin D1 is overexpressed in atypical teratoid/rhabdoid tumor with hSNF5/INI1 gene inactivation. $J$ Neurooncol 2005;73:117-24

20. Cheng L, Wu Q, Huang Z, Guryanova OA, Huang Q, Shou W, et al. L1CAM regulates DNA damage checkpoint response of glioblastoma stem cells through NBS1. EMBO J. 2011;30:800-13.

21. Held-Feindt J, Schmelz S, Hattermann K, Mentlein R, Mehdorn $\mathrm{HM}$, Sebens S. The neural adhesion molecule L1CAM confers chemoresistance in human glioblastomas. Neurochem Int. 2012;61:1183-91.

22. Mohanan V, Temburni MK, Kappes JC, Galileo DS. L1CAM stimulates glioma cell motility and proliferation through the fibroblast growth factor receptor. Clin Exp Metastasis. 2013;30:507-20.

23. Altevogt P, Doberstein K, Fogel M. L1CAM in human cancer. Int J Cancer. 2016;138:1565-76.

24. Fu Z, Tindall DJ. FOXOs, cancer and regulation of apoptosis. Oncogene. 2008;27:2312-9.

25. Amatu A, Sartore-Bianchi A, Siena S. NTRK gene fusions as novel targets of cancer therapy across multiple tumour types. ESMO Open. 2016;1:e000023.

26. Laetsch TW, DuBois SG, Mascarenhas L, Turpin B, Federman N, Albert CM, et al. Larotrectinib for paediatric solid tumours harbouring NTRK gene fusions: phase 1 results from a multicentre, open-label, phase 1/2 study. Lancet Oncol. 2018;19:705-14.

27. Drilon A, Laetsch TW, Kummar S, DuBois SG, Lassen UN, Demetri GD, et al. Efficacy of larotrectinib in TRK fusion-positive cancers in adults and children. N Engl J Med. 2018;378:731-9.

28. Yeh I, Tee MK, Botton T, Shain AH, Sparatta AJ, Gagnon A, et al. NTRK3 kinase fusions in Spitz tumours. J Pathol. 2016;240:282-90.

29. Wang L, Busam KJ, Benayed R, Cimera R, Wang J, Denley R, et al. Identification of NTRK3 fusions in childhood melanocytic neoplasms. J Mol Diagn. 2017;19:387-96.

30. Isales MC, Mohan LS, Quan VL, Garfield EM, Zhang B, Shi K, et al. Distinct genomic patterns in pigmented epithelioid 
melanocytoma: a molecular and histologic analysis of 16 cases. Am J Surg Pathol. 2019;43:480-8.

31. Yearley JH, Gibson C, Yu N, Moon C, Murphy E, Juco J, et al. PD-L2 expression in human tumors: relevance to anti-PD-1 therapy in cancer. Clin Cancer Res. 2017;23:3158-67.

32. Ross JS, Wang K, Chmielecki J, Gay L, Johnson A, Chudnovsky $\mathrm{J}$, et al. The distribution of BRAF gene fusions in solid tumors and response to targeted therapy. Int J Cancer. 2016;138:881-90.

33. Cordioli MI, Moraes L, Carvalheira G, Sisdelli L, Alves MT, Delcelo R, et al. AGK-BRAF gene fusion is a recurrent event in sporadic pediatric thyroid carcinoma. Cancer Med. 2016;5:1535-41.

34. Botton T, Yeh I, Nelson T, Vemula SS, Sparatta A, Garrido MC, et al. Recurrent BRAF kinase fusions in melanocytic tumors offer an opportunity for targeted therapy. Pigment Cell Melanoma Res. 2013;26:845-51.

35. Cuevas-Ocampo AK, Bollen AW, Goode B, Pajtler KW, Chavez L, Sharma T, et al. Genetic confirmation that ependymoma can arise as part of multiple endocrine neoplasia type 1 (MEN1) syndrome. Acta Neuropathol. 2017;133:661-3.

36. Heppner C, Bilimoria KY, Agarwal SK, Kester M, Whitty LJ, Guru SC, et al. The tumor suppressor protein menin interacts with NF-kappaB proteins and inhibits NF-kappaB-mediated transactivation. Oncogene. 2001;20:4917-25.

37. Gang D, Hongwei H, Hedai L, Ming Z, Qian H, Zhijun L. The tumor suppressor protein menin inhibits NF-kappaB-mediated transactivation through recruitment of Sirt1 in hepatocellular carcinoma. Mol Biol Rep. 2013;40:2461-6. 